\title{
Hopeafuran and a $C$-Glucosyl Resveratrol Isolated from Stem Wood of Hopea utilis
}

\author{
Toshiyuki Tanaka, ${ }^{*}, a$ Tetsuro Ito, ${ }^{a}$ Yoshimi Ido, ${ }^{a}$ Ken-ichi Nakaya, ${ }^{a}$ Munekazu Inuma, ${ }^{a}$ and \\ Veliah ChelladuraI $^{b}$ \\ Gifu Prefectural Institute of Health and Environmental Sciences, ${ }^{a}$ 1-1 Naka-fudogaoka, Kakamigahara 504-0838, Japan \\ and Survey of Medicinal Plant Unit, Central Council for Research in Ayurveda and Siddha, ${ }^{b}$ Tirunelveli 627002, Tamil \\ Nada, India. $\quad$ Received February 2, 2001; accepted March 14, 2001
}

\begin{abstract}
A new resveratrol dimer and a new $C$-glucosyl resveratrol were isolated from stem wood of Hopea utilis along with nine stilbenoid derivatives comprising bergenin and (+)-lyoniresinol. The structures have been elucidated on the basis of the spectroscopic evidence.
\end{abstract}

Key words Hopea utilis; Dipterocarpaceae; stilbenoid; hopeafuran; resveratrol-10-C-glucopyranoside; structure elucidation

Some of stilbene derivatives have been found to show various bioactivities, e.g. chemopreventive, ${ }^{1)}$ anti-inflammatory activity, ${ }^{2}$ inhibitory activity of histamine release ${ }^{3)}$ and gastric ATPase. ${ }^{4)}$ Dipterocarpaceaous plants generally contain stilbene derivatives, which consist of a resveratrol $\left(E-3,5,4^{\prime}\right.$-trihydroxystilbene) unit and are sometimes substituted with a glycosyl moiety. ${ }^{5,6)}$ We previously reported the isolation and structure elucidation of resveratrol oligomers in this family $\left(\right.$ Hopea ${ }^{7}{ }^{7}$ Vatica ${ }^{8,9)}$ and Shorea ${ }^{10,11)}$ ) and their distinctive cytotoxicity was disclosed. ${ }^{12)}$ The phytochemical constituents of Hopea utilis were examined in our continuing phytochemical studies of the Dipterocarpaceae oriented toward searching for leads with bioactivity. We report here the isolation and characterization of two new stilbenoids and 11 known compounds in H. utilis (BEDD.) BoLE.

Compound 1 (hopeafuran), $[\alpha]_{\mathrm{D}}-46^{\circ} \mathrm{C}$, a yellow solid, showed positive reaction to the Gibbs reagent. The negative ion FAB-MS exhibited an $[\mathrm{M}-\mathrm{H}]^{-}$ion peak at $m / z 465$ indicating the molecular weight to be 466 . The high-resolution (HR) negative FAB-MS at $\mathrm{m} / z 465.0974$ showed that the molecular formula is corresponding to $\mathrm{C}_{28} \mathrm{H}_{18} \mathrm{O}_{7}$. An absorption band in the IR spectrum $\left(1653 \mathrm{~cm}^{-1}\right)$ and a signal in the ${ }^{13} \mathrm{C}-\mathrm{NMR}$ spectrum $(\delta$ 196.4) showed the presence of a carbonyl group in the molecule. Usual methylation of 1 afforded a pentamethyl ether (1a), suggesting that $\mathbf{1}$ has five phenolic hydroxyl groups. Thus the remaining oxygen is attributable to an ether linkage in $\mathbf{1}$. The analysis of ${ }^{1} \mathrm{H}-\mathrm{NMR}$ spectrum (Table 1) exhibited the presence of two sets of ortho-coupled protons assignable to two 4-hydroxyphenyl groups $[\delta 7.70$
$(2 \mathrm{H}, \mathrm{d}, J=8.8 \mathrm{~Hz}, \mathrm{H}-2 \mathrm{a}, 6 \mathrm{a}), 6.98(2 \mathrm{H}, \mathrm{d}, J=8.8 \mathrm{~Hz}, \mathrm{H}-3 \mathrm{a}$, $5 \mathrm{a}) ; \delta 6.85(2 \mathrm{H}, \mathrm{dd}, J=8.3,1.3 \mathrm{~Hz}, \mathrm{H}-2 \mathrm{~b}, 6 \mathrm{~b}), 6.55(2 \mathrm{H}, \mathrm{d}$, $J=8.3 \mathrm{~Hz}, \mathrm{H}-3 \mathrm{~b}, 5 \mathrm{~b})]$ and two sets of meta-coupled aromatic protons on a $1,2,3,5$-tetrasubstituted benzene ring $[\delta 6.57$ $(1 \mathrm{H}, \mathrm{d}, J=2.6 \mathrm{~Hz}, \mathrm{H}-12 \mathrm{a}), 6.70(1 \mathrm{H}, \mathrm{d}, J=2.6 \mathrm{~Hz}, \mathrm{H}-14 \mathrm{a}) ; \delta$ $7.04(1 \mathrm{H}, \mathrm{d}, J=2.1 \mathrm{~Hz}, \mathrm{H}-12 \mathrm{~b}), 7.34(1 \mathrm{H}, \mathrm{d}, J=2.1 \mathrm{~Hz}, \mathrm{H}-$ $14 \mathrm{~b})]$. The presence of five phenolic hydroxyl groups $[\delta$ $8.00,8.36,8.80(2 \times \mathrm{OH}), 8.92]$ was also supported by the spectrum. A benzylic methine proton was observed as a signal in a high shifted-field $[\delta 6.12(1 \mathrm{H}, \mathrm{brs}, \mathrm{H}-7 \mathrm{~b})]$ and showed a cross peak with the aromatic protons $[\mathrm{H}-2 \mathrm{~b}(6 \mathrm{~b})]$ in the ${ }^{1} \mathrm{H}-{ }^{1} \mathrm{H}$ shift correlation spectroscopy spectrum. In the ${ }^{13} \mathrm{C}$-NMR spectrum (Table 2), 24 aromatic carbons and a methine carbon were assigned by the ${ }^{13} \mathrm{C}-{ }^{1} \mathrm{H}$ shift correlation spectroscopy and correlation spectroscopy involving longrange coupling (COLOC) spectrum $(J=8,10 \mathrm{~Hz})$ (Fig. 2). The remaining carbon signals $[\delta 153.3(\mathrm{C}-7 \mathrm{a}), \delta 116.4(\mathrm{C}-$ 8a)] were observed as quaternary olefinic carbons, and the behavior of chemical shift was closely similar to those of a benzofuran moiety of malibatol $\mathrm{A}^{13)}(\delta 150.6, \delta 117.4$, Table 2). In the COLOC spectrum (Fig. 2), significant ${ }^{3} J$ longrange correlations were observed between $\mathrm{C}-7 \mathrm{a} / \mathrm{H}-2 \mathrm{a}(6 \mathrm{a})$, C-8a/H-14a, C-9a/H-7b, C-11a/H-7b and C-2b(6b)/H-7b, showing that a partial structure of 1 (ring- $\mathrm{A}_{1}-\mathrm{C}-7 \mathrm{a}-\mathrm{C}-8 \mathrm{a}-$ ring- $\mathrm{A}_{2}-\mathrm{C}-7 \mathrm{~b}$-ring $\mathrm{B}_{1}$ ) was the same as that of malibatol $\mathrm{A}$. On the other hand, the carbonyl carbon signal (C-8b) displayed cross peaks with the aromatic proton (H-14b) and the aliphatic methine proton $(\mathrm{H}-7 \mathrm{~b})$, indicating that the carbonyl group was attached to $\mathrm{C}-9 \mathrm{~b}$ and $\mathrm{C}-7 \mathrm{~b}$. Considering the mole-

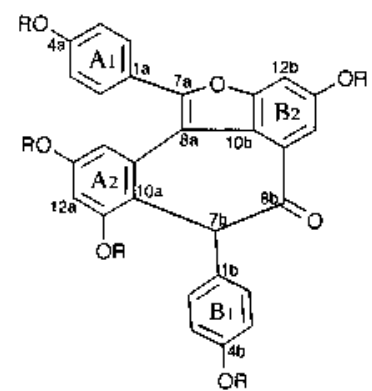

$1 \mathrm{R}=\mathrm{H}$

1a $\mathrm{R}=\mathrm{Me}$<smiles>Oc1ccc(C=Cc2cc(O)cc(O)c2F)cc1</smiles>

$\begin{array}{ll}2 & R=g l c \\ 3 & R=H\end{array}$

Fig. 1<smiles>Oc1ccc(-c2oc3cc(O)cc4c3c2-c2c(O)cc(O)cc2C4c2ccc(O)cc2)cc1</smiles>

8 
Table 1. ${ }^{1} \mathrm{H}-\mathrm{NMR}$ Spectral Data of $(300 \mathrm{MHz}) \mathbf{1}, \mathbf{1 a}, \mathbf{8}$ and $\mathbf{8 a}$

\begin{tabular}{|c|c|c|c|c|}
\hline \multirow{2}{*}{ No. } & \multicolumn{4}{|c|}{$\delta_{\mathrm{H}}$} \\
\hline & $\mathbf{1}^{a)}$ & $1 \mathbf{a}^{b)}$ & $\mathbf{8}^{a)}$ & $8 \mathbf{a}^{b)}$ \\
\hline 2a. $6 \mathrm{a}$ & $7.70(2 \mathrm{H}, \mathrm{d}, 8.8)$ & $7.75(2 \mathrm{H}, \mathrm{d}, 8.8)$ & $7.54(2 \mathrm{H}, \mathrm{d}, 8.8)$ & $7.61(2 \mathrm{H}, \mathrm{d}, 8.8)$ \\
\hline $3 a, 5 a$ & $6.98(2 \mathrm{H}, \mathrm{d}, 8.8)$ & $6.97(2 \mathrm{H}, \mathrm{d}, 8.8)$ & $6.92(2 \mathrm{H}, \mathrm{d}, 8.8)$ & $6.93(2 \mathrm{H}, \mathrm{d}, 8.8)$ \\
\hline $12 \mathrm{a}$ & $6.57(1 \mathrm{H}, \mathrm{d}, 2.6)$ & $6.50(1 \mathrm{H}, \mathrm{d}, 2.6)$ & $6.49(1 \mathrm{H}, \mathrm{d}, 2.4)$ & $6.41(1 \mathrm{H}, \mathrm{d}, 2.4)$ \\
\hline $14 \mathrm{a}$ & $6.70(1 \mathrm{H}, \mathrm{d}, 2.6)$ & $6.75(1 \mathrm{H}, \mathrm{d}, 2.6)$ & $6.66(1 \mathrm{H}, \mathrm{d}, 2.4)$ & $6.71(1 \mathrm{H}, \mathrm{d}, 2.4)$ \\
\hline $2 \mathrm{~b}, 6 \mathrm{~b}$ & $6.85(2 \mathrm{H}, \mathrm{dd}, 8.3,1.3)$ & $6.87(2 \mathrm{H}, \mathrm{dd}, 8.3,1.3)$ & $7.20(2 \mathrm{H}, \mathrm{d}, 8.6)$ & $7.11(2 \mathrm{H}, \mathrm{d}, 8.6)$ \\
\hline $3 b, 5 b$ & $6.55(2 \mathrm{H}, \mathrm{d}, 8.3)$ & $6.55(2 \mathrm{H}, \mathrm{d}, 8.3)$ & $6.45(2 \mathrm{H}, \mathrm{d}, 8.6)$ & $6.51(2 \mathrm{H}, \mathrm{d}, 8.6)$ \\
\hline $7 \mathrm{~b}$ & $6.12(1 \mathrm{H}, \mathrm{brs})$ & $6.20(1 \mathrm{H}, \mathrm{brs})$ & $5.59(1 \mathrm{H}, \mathrm{brs})$ & $5.57(1 \mathrm{H}, \mathrm{brd})$ \\
\hline $8 b$ & & & $5.43(1 \mathrm{H}, \mathrm{brs})$ & $5.40(1 \mathrm{H}, \mathrm{brd})$ \\
\hline $12 b$ & $7.04(1 \mathrm{H}, \mathrm{d}, 2.1)$ & $7.05(1 \mathrm{H}, \mathrm{d}, 2.1)$ & $6.67(1 \mathrm{H}, \mathrm{d}, 2.0)$ & $6.78(1 \mathrm{H}, \mathrm{d}, 2.0)$ \\
\hline $14 \mathrm{~b}$ & $7.34(1 \mathrm{H}, \mathrm{d}, 2.1)$ & $7.44(1 \mathrm{H}, \mathrm{d}, 2.1)$ & $7.22(1 \mathrm{H}, \mathrm{d}, 2.0)$ & $7.12(1 \mathrm{H}, \mathrm{d}, 2.0)$ \\
\hline $\mathrm{OH}-8 \mathrm{~b}$ & & & $5.35(1 \mathrm{H}, \mathrm{brd})$ & $4.89(1 \mathrm{H}, \mathrm{brs})$ \\
\hline \multirow[t]{5}{*}{$\mathrm{Ar}-\mathrm{OH}(\mathrm{OMe})$} & $8.92(1 \mathrm{H}, \mathrm{br} \mathrm{s}, \mathrm{OH}-4 \mathrm{a})$ & 3.89 (3H, s, OMe-4a) & $7.81-8.46(5 \mathrm{H}, \mathrm{brs})$ & $3.84(3 \mathrm{H}, \mathrm{s}, \mathrm{OMe}-4 \mathrm{a})$ \\
\hline & $8.80(1 \mathrm{H}, \mathrm{br} \mathrm{s}, \mathrm{OH}-11 \mathrm{a})$ & $3.85(3 \mathrm{H}, \mathrm{s}, \mathrm{OMe}-11 \mathrm{a})$ & & $3.81(3 \mathrm{H}, \mathrm{s}, \mathrm{OMe}-11 \mathrm{a})$ \\
\hline & $8.36(1 \mathrm{H}, \mathrm{brs}, \mathrm{OH}-13 \mathrm{a})$ & $3.52(3 \mathrm{H}, \mathrm{s}, \mathrm{OMe}-13 \mathrm{a})$ & & $3.46(3 \mathrm{H}, \mathrm{s}, \mathrm{OMe}-13 \mathrm{a})$ \\
\hline & $8.00(1 \mathrm{H}, \mathrm{br} \mathrm{s}, \mathrm{OH}-4 \mathrm{~b})$ & $3.60(3 \mathrm{H}, \mathrm{s}, \mathrm{OMe}-4 \mathrm{~b})$ & & $3.60(3 \mathrm{H}, \mathrm{s}, \mathrm{OMe}-4 \mathrm{~b})$ \\
\hline & $8.80(1 \mathrm{H}, \mathrm{br} \mathrm{s}, \mathrm{OH}-13 \mathrm{~b})$ & $3.86(3 \mathrm{H}, \mathrm{s}, \mathrm{OMe}-13 \mathrm{~b})$ & & $3.82(3 \mathrm{H}, \mathrm{s}, \mathrm{OMe}-13 \mathrm{~b})$ \\
\hline
\end{tabular}

a) Measured in $\mathrm{CD}_{3} \mathrm{COCD}_{3}$. b) Measured in $\mathrm{CDCl}_{3}$. All protons were assigned by ${ }^{1} \mathrm{H}-{ }^{1} \mathrm{H},{ }^{1} \mathrm{H}-{ }^{1} \mathrm{H}$ long range, ${ }^{13} \mathrm{C}-{ }^{1} \mathrm{H}$ COSY, COLOC and $\mathrm{HMBC}$ spectrum.

Table 2. ${ }^{13} \mathrm{C}-\mathrm{NMR}$ Spectral Data (75 MHz) of 1, 1a, 8 and $\mathbf{8 a}$

\begin{tabular}{|c|c|c|c|c|}
\hline \multirow{2}{*}{ No. } & \multicolumn{4}{|c|}{$\delta_{\mathrm{C}}$} \\
\hline & $\mathbf{1}^{a)}$ & $1 a^{b)}$ & $\mathbf{8}^{a)}$ & $\mathbf{8 a}^{b)}$ \\
\hline 1a & 123.1 & 123.2 & 124.4 & 124.3 \\
\hline $2 \mathrm{a}, 6 \mathrm{a}$ & 131.0 & 130.2 & 130.8 & 130.2 \\
\hline $3 \mathrm{a}, 5 \mathrm{a}$ & 116.7 & 114.2 & 116.4 & 113.9 \\
\hline $4 \mathrm{a}$ & 159.5 & 160.6 & 158.6 & 160.0 \\
\hline $7 \mathrm{a}$ & 153.3 & 152.9 & 150.8 & 150.8 \\
\hline $8 \mathrm{a}$ & 116.4 & 116.2 & 117.1 & 116.3 \\
\hline $9 \mathrm{a}$ & 135.3 & 134.1 & 135.6 & 134.1 \\
\hline $10 \mathrm{a}$ & 114.1 & 115.8 & 120.5 & 121.9 \\
\hline $11 \mathrm{a}$ & 158.3 & 159.7 & 157.0 & 158.5 \\
\hline $12 \mathrm{a}$ & 103.1 & 98.6 & 102.4 & 98.0 \\
\hline $13 \mathrm{a}$ & 157.7 & 159.0 & 156.7 & 158.2 \\
\hline $14 \mathrm{a}$ & 109.0 & 105.5 & 109.7 & 106.0 \\
\hline $1 \mathrm{~b}$ & 131.1 & 130.5 & 133.0 & 131.7 \\
\hline $2 b, 6 b$ & 128.5 & 127.6 & 130.4 & 129.5 \\
\hline $3 b, 5 b$ & 115.7 & 113.3 & 114.8 & 113.0 \\
\hline $4 \mathrm{~b}$ & 156.4 & 157.7 & 155.5 & 157.4 \\
\hline $7 b$ & 56.1 & 56.2 & 48.4 & 47.6 \\
\hline $8 \mathrm{~b}$ & 196.4 & 196.0 & 74.4 & 73.5 \\
\hline $9 b$ & 129.9 & 129.9 & 139.7 & 137.2 \\
\hline $10 \mathrm{~b}$ & 122.5 & 122.4 & 118.5 & 117.9 \\
\hline $11 \mathrm{~b}$ & 154.9 & 154.0 & 154.7 & 153.9 \\
\hline $12 \mathrm{~b}$ & 102.5 & 101.2 & 95.9 & 93.8 \\
\hline $13 \mathrm{~b}$ & 156.1 & 157.6 & 156.1 & 158.1 \\
\hline $14 \mathrm{~b}$ & 112.0 & 109.7 & 110.2 & 108.2 \\
\hline \multirow[t]{5}{*}{$\mathrm{OMe}$} & \multicolumn{3}{|c|}{$55.0(3 \times \mathrm{C})$} & $55.1(\mathrm{C}-4 \mathrm{a})$ \\
\hline & \multicolumn{3}{|c|}{55.4} & $55.6(\mathrm{C}-11 \mathrm{a})$ \\
\hline & \multirow{3}{*}{\multicolumn{3}{|c|}{56.1}} & $54.9(\mathrm{C}-13 \mathrm{a})^{c)}$ \\
\hline & & & & $54.9(\mathrm{C}-4 \mathrm{~b})^{c)}$ \\
\hline & & & & $54.9(\mathrm{C}-13 \mathrm{~b})^{c)}$ \\
\hline
\end{tabular}

a) Measured in $\mathrm{CD}_{3} \mathrm{COCD}_{3}$. b) Measured in $\mathrm{CDCl}_{3}$. c) Overlapping. All carbons were assigned by ${ }^{13} \mathrm{C}-{ }^{1} \mathrm{H}$ COSY, COLOC and HMBC spectrum.

cular weight, the structure of $\mathbf{1}$ was characterized as shown in Fig. 2, where the benzofuran ring was fused to ring $B_{2}$. The benzyl methine proton $(\mathrm{H}-7 \mathrm{~b})$ appears in a lower field than that of $\mathbf{8}$, which could be explained by the deshielding effect which is caused by the carbonyl group. Stilbenoids having benzofuran moiety such as hopeafuran have been isolated from Vitis vinifera, ${ }^{14)}$ Hopea malbato ${ }^{13)}$ and Shorea sp.

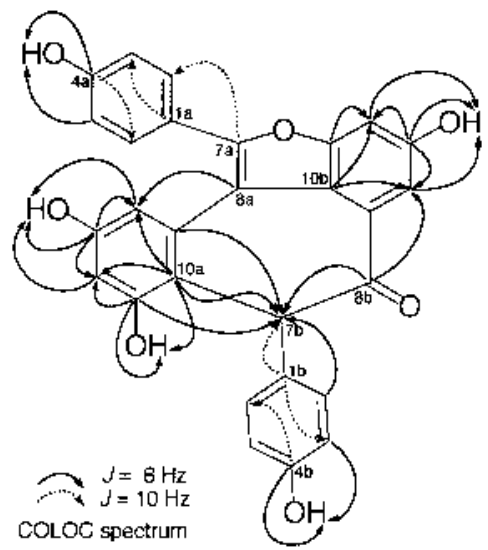

Fig. 2. Correlations Observed in the COLOC Spectrum of $\mathbf{1}$

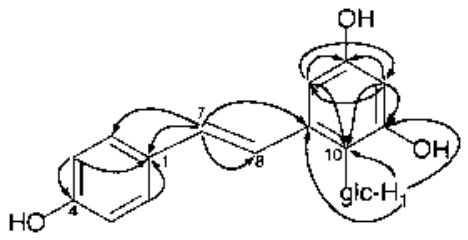

Fig. 3. HMBC Spectrum of 2

(Dipterocarpaceae). ${ }^{15)}$

Compound 2 was positive to the Gibbs reagent and had the molecular formula of $\mathrm{C}_{20} \mathrm{H}_{22} \mathrm{O}_{8}$, supported by the HR-FABMS ( $m / z$ 389.1244). The ${ }^{1} \mathrm{H}-\mathrm{NMR}$ spectrum showed the signals due to a 4-hydroxyphenyl group $[\delta 7.36(2 \mathrm{H}, \mathrm{d}, J=8.5$ $\mathrm{Hz}, \mathrm{H}-2,6), 6.79(2 \mathrm{H}, \mathrm{d}, J=8.5 \mathrm{~Hz}, \mathrm{H}-3,5)]$ and a set of meta-coupled aromatic protons on a 1,2,3,5-tetrasubstituted benzene ring $[\delta 6.22(1 \mathrm{H}, \mathrm{d}, J=2.4 \mathrm{~Hz}, \mathrm{H}-12), 6.56(1 \mathrm{H}, \mathrm{d}$, $J=2.4 \mathrm{~Hz}, \mathrm{H}-14)]$. Trans geometry of two olefinic protons $[\delta$ $6.76(1 \mathrm{H}, \mathrm{d}, J=16.3 \mathrm{~Hz}, \mathrm{H}-7), 7.48(1 \mathrm{H}, \mathrm{brd}, J=16.3 \mathrm{~Hz}, \mathrm{H}-$ $8)$ ] were also exhibited in the spectrum. The ${ }^{1} \mathrm{H}$ - and ${ }^{13} \mathrm{C}$ NMR spectral data showed the presence of a $C$-glucopyranosyl moiety $\left[\delta_{\mathrm{H}} 4.92(1 \mathrm{H}, \mathrm{d}, J=9.6 \mathrm{~Hz}) ; \delta_{\mathrm{C}} 81.7,79.5,78.5\right.$, $74.4,70.9,61.9] .^{10,11)}$ In the heteronuclear multiple bond connectivity (HMBC) spectrum (Fig. 3), distinct correlations 
were observed between the olefinic proton (H-7) and aromatic carbons [C-2(6), C-9], suggesting that the aglycone moiety was resveratrol. The location of the $C$-glucosyl moiety was determined to be at $\mathrm{C}-10$ by the HMBC spectrum which displayed the cross peak between the anomeric proton $(\delta 4.92)$ and the three aromatic carbons at C-9 $(\delta 140.7)$, C$10(\delta 114.7)$ and $\mathrm{C}-11(\delta 158.5)$. Therefore, the structure of 2 was concluded to be 10-C- $\beta$-glucopyranosylresveratrol.

Eight known stilbenoids, resveratrol (3), dihydroresveratrol (4), ${ }^{16)}(-)$ - $\varepsilon$-viniferin $(5),{ }^{13)}(+)$-ampelopsin A (6), ${ }^{17)}$ (-)-balanocarpol (7), ${ }^{18)}$ malibatol A (8), ${ }^{13)}$ vaticanol B $(\mathbf{1 1}){ }^{8)}(-)$-hopeaphenol $(\mathbf{1 2})^{19)}$ and $(+)$-isohopeaphenol $(\mathbf{1 3})^{19)}$ were identified in addition to bergenin $(9)^{20)}$ and $(+)-$ lyoniresinol $(\mathbf{1 0})^{21)}$ by the analysis of spectral data and by comparison with authentic samples. Isolation and structure elucidation of other stilbenoids in acetone and $\mathrm{MeOH}$ extracts is now in progress.

\section{Experimental}

${ }^{1} \mathrm{H}$ - and ${ }^{13} \mathrm{C}-\mathrm{NMR}$ spectra were recorded on JNM EX-400 and LA-300 (JEOL) spectrometers. Chemical shifts are shown as $\delta$ values with tetramethylsilane (TMS) as an internal reference. Peak multiplicities are quoted in Hz. Negative ion FAB-MS was measured on a JMS-DX-300 spectrometer equipped with a JMA 3500 data analysis system (JEOL). UV spectra were recorded on a UV-2200 spectrometer (Shimadzu), IR spectra on a FT-IR8000 spectrometer (JASCO), and optical rotations on a P-1020 (JASCO) polarimeter. Silica gel 60 ( $70-230$ mesh, Merck) and Sephadex LH-20 (Pharmacia) were used for column chromatography; silica gel 60H (Merck) was used for vacuum liquid chromatography (VLC); Kiesel-gel $60 \mathrm{~F}_{254}$ (Merck) was used as analytical and preparative TLC.

Extraction and Isolation of Compounds (1-13) Dried stem wood of Hopea utilis $(1.0 \mathrm{~kg}$ ) collected in India in October, 1999 was powdered and extracted successively with acetone $(31 \times 3), \mathrm{MeOH}(31 \times 3)$ and $70 \% \mathrm{MeOH}$ $(31 \times 3)$ at room temperature. A part $(160 \mathrm{~g})$ of the acetone extract $(180 \mathrm{~g})$ was chromatographed on $\mathrm{Si}$ gel $(1200 \mathrm{~g})$ eluted with $\mathrm{CHCl}_{3}-\mathrm{MeOH}$ mixtures to divide into 17 fractions (Fr. 1-17). Compounds $3(12 \mathrm{mg}), 4(6 \mathrm{mg})$ and $10(21 \mathrm{mg})$ were obtained from $\mathrm{Fr} .7\left[\mathrm{CHCl}_{3}-\mathrm{MeOH}(10: 1)\right.$ fraction] after purification by Sephadex LH-20 (acetone) and preparative TLC (EtOAc$\left.\mathrm{CHCl}_{3}-\mathrm{MeOH}-\mathrm{H}_{2} \mathrm{O}=80: 40: 11: 2\right)$. Fraction $9\left[\mathrm{CHCl}_{3}-\mathrm{MeOH}(10: 1)\right.$ fraction] was further chromatographed by Sephadex LH-20 using MeOH to give 10 fractions. Compound $\mathbf{9}(1.8 \mathrm{~g})$ was obtained in a pure form after recrystallization (acetone) from the third fraction. The sixth fraction afforded $1(65 \mathrm{mg})$ and $\mathbf{5}(250 \mathrm{mg})$ after purification by Sephadex LH-20 column [acetone- $\mathrm{MeOH}(5: 1)]$. Fraction 13 [ $\mathrm{CHCl}_{3}-\mathrm{MeOH}(6: 1)$ fraction] was repeatedly chromatographed by Sephadex $\mathrm{LH}-20[\mathrm{MeOH}$ and acetone- $\mathrm{MeOH}$ (5:1)] to isolate $8(80 \mathrm{mg}), \mathbf{6}(85 \mathrm{mg})$ and $\mathbf{7}(1.3 \mathrm{~g})$. Compounds $\mathbf{2}(12 \mathrm{mg})$, $11(2.3 \mathrm{~g}), \mathbf{1 2}(3.6 \mathrm{~g})$ and $\mathbf{1 3}(9 \mathrm{mg})$ were obtained from Fr. $16\left[\mathrm{CHCl}_{3}-\right.$ $\mathrm{MeOH}(6: 1)$ fraction] after purification by Sephadex LH-20 (MeOH) and preparative TLC (EtOAc- $\left.\mathrm{CHCl}_{3}-\mathrm{MeOH}-\mathrm{H}_{2} \mathrm{O}=15: 8: 4: 1\right)$.

Compound 1 (Hopeafuran): A yellow solid. Negative ion HR-FAB-MS: $\left([\mathrm{M}-\mathrm{H}]^{-}\right) \mathrm{m} / z$ 465.0961 (Calcd 465.0974 for $\mathrm{C}_{28} \mathrm{H}_{17} \mathrm{O}_{7}$ ); Negative ion FABMS: $m / z 465\left([\mathrm{M}-\mathrm{H}]^{-}\right)$. UV $\lambda_{\max }(\mathrm{MeOH}) \mathrm{nm}: 212,255 \mathrm{sh}, 297,396$. IR $\lambda$ $(\mathrm{KBr}) \mathrm{cm}^{-1}: 3384,1653,1613,1512 .[\alpha]_{\mathrm{D}}^{24}-46^{\circ}(c=0.1, \mathrm{MeOH})$. The ${ }^{1} \mathrm{H}-$ and ${ }^{13} \mathrm{C}$-NMR spectral data are listed in Tables 1 and 2, respectively.

Methylation of 1 Compound $1(20 \mathrm{mg})$ was allowed to react with $\mathrm{K}_{2} \mathrm{CO}_{3}(2 \mathrm{~g})$ and $\mathrm{MeI}(0.5 \mathrm{~g})$ in dry acetone under reflux for $6 \mathrm{~h}$. The crude product $(22 \mathrm{mg})$ was purified by preparative TLC $[n$-hexane-EtOAc $(1: 1)]$ to afford 1a as an amorphous yellow solid (15 mg). The ${ }^{1} \mathrm{H}-$ and ${ }^{13} \mathrm{C}-\mathrm{NMR}$ spectral data are listed in Tables 1 and 2, respectively.

Compound 2 (Resveratrol-10- $C$ - $\beta$-glucopyranoside): A white amorphous powder. Negative ion HR-FAB-MS: $\left([\mathrm{M}-\mathrm{H}]^{-}\right) \mathrm{m} / \mathrm{z} 389.1244$ (Calcd 389.1236 for $\mathrm{C}_{20} \mathrm{H}_{21} \mathrm{O}_{8}$ ); Negative ion FAB-MS: $m / z 389\left([\mathrm{M}-\mathrm{H}]^{-}\right)$. UV $\lambda_{\max }(\mathrm{MeOH}) \mathrm{nm}: 212,293 .[\alpha]_{\mathrm{D}}^{24}+23^{\circ}(c=0.1, \mathrm{MeOH}) .{ }^{1} \mathrm{H}-\mathrm{NMR}(300$ MHz, acetone- $\left.d_{6}\right) \delta: 7.36(2 \mathrm{H}, \mathrm{d}, J=8.5 \mathrm{~Hz}, \mathrm{H}-2,6), 6.79(2 \mathrm{H}, \mathrm{d}, J=8.5 \mathrm{~Hz}$, $\mathrm{H}-3,5), 6.76(1 \mathrm{H}, \mathrm{d}, J=16.3 \mathrm{~Hz}, \mathrm{H}-7), 7.48(1 \mathrm{H}, \mathrm{br} \mathrm{d}, \mathrm{H}-8), 6.22(1 \mathrm{H}, \mathrm{d}$, $J=2.4 \mathrm{~Hz}, \mathrm{H}-12), 6.56(1 \mathrm{H}, \mathrm{d}, J=2.4 \mathrm{~Hz}, \mathrm{H}-14), 4.92(1 \mathrm{H}, \mathrm{d}, J=9.6 \mathrm{~Hz}, \mathrm{H}-$ glc-1), $3.71(1 \mathrm{H}, \mathrm{t}, J=9.6 \mathrm{~Hz}, \mathrm{H}-\mathrm{glc}-2), 3.58(1 \mathrm{H}, \mathrm{t}, J=9.6 \mathrm{~Hz}, \mathrm{H}-\mathrm{glc}-3)$, $3.67(1 \mathrm{H}, \mathrm{t}, J=9.6 \mathrm{~Hz}, \mathrm{H}$-glc- 4$), 3.44(1 \mathrm{H}, \mathrm{dd}, J=9.6,3.5 \mathrm{~Hz}, \mathrm{H}$-glc-5), 3.81 $\left(2 \mathrm{H}, \mathrm{m}, \mathrm{H}\right.$-glc-6); ${ }^{13} \mathrm{C}-\mathrm{NMR}\left[75 \mathrm{MHz}\right.$, acetone- $\left.d_{6}\right) \delta: 130.4(\mathrm{C}-1), 128.8(\mathrm{C}-$ 2, 6), 116.4 (C-3, 5), 158.1 (C-4), 131.0 (C-7), 126.0 (C-8), 140.7 (C-9), 114.7 (C-10), 158.5 (C-11), 104.0 (C-12), 158.5 (C-13), 105.7 (C-14), 78.5 (C-glc-1), 74.4 (C-glc-2), 79.5 (C-glc-3), 70.9 (C-glc-4), 81.7 (C-glc-5), 61.9 (C-glc-6).

\section{References}

1) Jang M., Cai L., Udeani G. O., Slowing K. V., Thomas C. F., Beecher C. W. W., Fong H. H. S., Farnsworth N. R., Kinghorn A. D., Mehta R. G., Moon R. C., Pezzuto J. M., Science, 275, 218 -220 (1997).

2) Kitanaka S., Ikezawa T., Yasukawa K., Yamanouchi S., Takido M., Sung H., Kim I., Chem. Pharm. Bull., 38, 432-435 (1990).

3) Inamori Y., Ogawa M., Tsujibo H., Baba K., Kozawa M., Nakamura H., Chem. Pharm. Bull., 39, 805-807 (1991).

4) Murakami S., Arai I., Muramatsu M., Otomo S., Baba K., Kido T., Kozawa M., Biochem. Pharm., 44, 33-37 (1992).

5) Gorham J., "The Biochemistry of the Stilbenoids," Chapman \& Hall, London, 1995.

6) Sotheeswaran S., Pasupathy V., Phytochemistry, 32, 1083-1092 (1993).

7) Tanaka T., Ito T., Ido Y., Son T.-K., Nakaya K., Iinuma M., Ohyama M., Chelladurai V., Phytochemistry, 53, 1015-1019 (2000).

8) Tanaka T., Ito T., Nakaya K., Iinuma M., Riswan S., Phytochemistry, 53, 63-69 (2000).

9) Tanaka T., Ito T., Nakaya K., Iinuma M., Takahashi Y., Naganawa H., Matsuura N., Ubukata M., Tetrahedron Lett., 41, 7929-7932 (2000).

10) Ito T., Tanaka T., Ido Y., Nakaya K., Iinuma M., Riswan S., Chem. Pharm. Bull., 48, 1001-1005 (2000).

11) Ito T., Tanaka T., Ido Y., Nakaya K., Iinuma M., Riswan S., Chem. Pharm. Bull., 48, 1959-1963 (2000).

12) Ohyama M., Tanaka T., Ito T., Iinuma M., Bastow K. F., Lee K., Bioorg. Med. Chem. Lett., 9, 3057-3060 (1999).

13) Dai J.-R., Hallock Y. F., Cardellina J. H., Boyd M. R., J. Nat. Prod., 61, 351-353 (1998).

14) Ito J., Takaya Y., Oshima Y., Niwa M., Tetrahedron, 55, 2529-2544 (1999).

15) Hirano Y., Seri K., Kondo R., Sakai K., Abstract Papers of the 46th Annual Meeting of the Japanese Society of Pharmacognosy 1999, Osaka, p. 108.

16) Elferaly F. S., J. Nat. Prod., 47, 89-92 (1984).

17) Oshima Y., Ueno Y., Hikino H., Tetrahedron, 46, 5121—5126 (1990).

18) Diyasena M. N. C., Sotheeswaran S., Surendrakumar S., Balasubramania S., Bokel M., Kraus W., J. Chem. Soc., Perkin Trans. I, 1985, 1807.

19) Ito J., Niwa M., Oshima Y., Heterocycles, 45, 1809-1813 (1997).

20) Taneyama M., Yoshida S., Kobayashi M., Hasegawa M., Phytochemistry, 22, 1053-1054 (1983).

21) Rowe J. W., Seikel K. M., Roy D. N., Jorgensen E., Phytochemistry, 11, 2513-2517 (1972). 